\title{
Improved bounce-back methods for no-slip walls in lattice-Boltzmann schemes: Theory and simulations
}

\author{
M. Rohde, ${ }^{*}$ D. Kandhai ${ }^{\dagger}$ J. J. Derksen, and H. E. A. Van den Akker \\ Kramers Laboratorium voor Fysische Technologie, Delft University of Technology, Prins Bernhardlaan 6, 2628 BW Delft, \\ The Netherlands \\ (Received 25 July 2002; revised manuscript received 3 February 2003; published 10 June 2003)
}

\begin{abstract}
A detailed analysis is presented for the accuracy of several bounce-back methods for imposing no-slip walls in lattice-Boltzmann schemes. By solving the lattice-BGK (Bhatnagar-Gross-Krook) equations analytically in the case of plane Poiseuille flow, it is found that the volumetric approach by Chen et al. is first-order accurate in space, and the method of Bouzidi et al. second-order accurate in space. The latter method, however, is not mass conservative because of errors associated with interpolation of densities residing on grid nodes. Therefore, similar interpolations are applied to Chen's volumetric scheme, which indeed improves the accuracy in the case of plane Poiseuille flow with boundaries parallel to the underlying grid. For skew boundaries, however, it is found that the accuracy remains first order. An alternative volumetric approach is proposed with a more accurate description of the geometrical surface. This scheme is demonstrated to be second-order accurate, even in the case of skew channels. The scheme is mass conservative in the propagation step because of its volumetric description, but still not in the collision step. However, the deviation in the mass is, in general, found to be small and proportional to the second-order terms in the standard BGK equilibrium distribution. Consequently, the scheme is a priori mass conservative for Stokes flow.
\end{abstract}

DOI: 10.1103/PhysRevE.67.066703 PACS number(s): 02.70. $-\mathrm{c}$, 05.20.Dd, 45.50. $-\mathrm{j}, 47.11 .+\mathrm{j}$

\section{INTRODUCTION}

In the last decade, lattice-gas and lattice-Boltzmann methods have proven to be versatile tools for simulating a wide variety of fluid dynamical applications [1]. These include turbulent single-phase flows, transport in porous media, and multiphase flows in several scientific and industrial applications [1-3]. An important issue encountered in all these simulations is the way boundary conditions are being imposed, in particular, at inflow, outflow, and no-slip boundaries.

In this respect, the well-known bounce-back scheme can be considered as an extremely simple method for dealing with arbitrary complex solid-fluid interfaces. Although still widely being applied, it is by now well accepted that the bounce-back scheme is not very accurate. Several numerical simulations confirm that the method is first-order accurate in space and although applied only at the boundaries, it also does affect the accuracy in the bulk of the fluid. Therefore, it degenerates practical simulations performed using latticeBoltzmann methods to first-order accuracy in space, although lattice-Boltzmann methods, in general, are secondorder accurate in space (see Ref. [4], and references therein).

In the recent few years, much effort has been spent in improving the bounce-back method. In a very early stage, several modifications have been proposed, which, however, were restricted to rather regular geometries, e.g., flat walls and octagonal cylinders [5-10]. More recently, sophisticated schemes have been proposed that are suitable for dealing with irregular geometries. The most notable contributions among these are the volumetric methods by Verberg and

\footnotetext{
*Electronic address: m.rohde@klft.tn.tudelft.nl

${ }^{\dagger}$ Electronic address: b.d.kandhai@klft.tn.tudelft.nl
}

Ladd [11] and Chen et al. [12] and the interpolation technique by Bouzidi et al. [13].

Though these methods can deal with complex boundaries, it is not obvious that second-order accuracy is automatically obtained. Verberg and Ladd showed that their method is second-order accurate in the case of boundaries parallel to the underlying grid, but reduces to first order for skew channels. The method of Bouzidi et al., on the other hand, is found to be second-order accurate in more complex cases such as flow around cylinders. A drawback of this scheme is that mass is not conserved, because of errors associated with interpolation of densities residing on grid nodes. With a volumetric approach, on the contrary, mass can be controlled, so that a mass conservative scheme can be set up [14].

In this paper, we therefore revisit the volumetric method by Chen et al. [12] and present a detailed analysis in the case of plane Poiseuille flow, following the work of He et al. [15]. An analytical expression for the error is derived and compared with the data obtained by numerical simulations. We also analyze the method of Bouzidi et al. [13] and include in our study the recently published method and analysis by Verberg and Ladd [11].

Inspired by the findings of these analyses, we propose two alternative volumetric methods with the intention to obtain second-order accuracy. These approaches are then analyzed theoretically and numerically in the case of plane Poiseuille flow for varying orientations of the boundaries with respect to the underlying grid.

\section{ANALYSIS OF EXISTING MODIFIED BOUNCE-BACK SCHEMES}

\section{A. Volumetric scheme of Chen et al.}

The lattice-Boltzmann method originally is a finitedifference discretization scheme [16]. It can, however, also 
be interpreted as a volumetric scheme. By definition, each grid node $\vec{x}$ is located in the center of a cubic cell (i.e., grid cell) with size $\Delta x=\Delta y=\Delta z$. If we assume that the mass, typically located on the grid node, is distributed uniformly throughout the cell, the traditional lattice-Boltzmann equation can be rewritten as follows:

$$
N_{i}\left(\vec{x}+\vec{c}_{i} \Delta t, t+\Delta t\right)=N_{i}(\vec{x}, t)+\Omega_{i}(\vec{N}(\vec{x}, t)) .
$$

The only difference between Eq. (1) and the traditional lattice-Boltzmann equation is the use of masses $N_{i}$ rather than densities $n_{i}$. As these masses reside in cubic cells with volume $\Delta V$, the density in a cubic cell is equal to $n_{i}$ $=N_{i} / \Delta V$. In the vicinity of a solid surface, however, this volumetric definition is different from the original scheme, because some cells may be cut by the surface and become noncubic.

Chen et al. [12] proposed a modified lattice-Boltzmann scheme for these cells, where solid surfaces are defined by facets $S^{\alpha}$ with area $A^{\alpha}$ and a surface normal $\vec{n}^{\alpha}$. During the propagation step, a fraction of the mass in the cells adjacent to the surface (i.e., boundary cells) hits the surface of an object, and is simply reflected (i.e., bounced back) in the opposite direction $i\left(\vec{c}_{i *} \equiv-\vec{c}_{i}\right)$. For this bounce-back process, a volumetric lattice-Boltzmann scheme for boundary cells can be set up as follows:

$$
N_{i}\left(\vec{x}+\vec{c}_{i} \Delta t, t+\Delta t\right)=P_{i}^{u n d i s t}(\vec{x}) N_{i}^{\prime}(\vec{x}, t)+Q_{i}\left(\vec{x}+\vec{c}_{i} \Delta t, t\right),
$$

where $N_{i}^{\prime}(\vec{x}, t) \equiv N_{i}(\vec{x}, t)+\Omega_{i}(\vec{x}, t)$ is the right-hand side of the lattice-Boltzmann scheme for a cubic cells and $P_{i}^{\text {undist }}(\vec{x})$ represents the fraction of mass that does not hit the surface during the propagation step. This fraction moves undisturbedly from cell $\vec{x}$ to cell $\vec{x}+\vec{c}_{i} \Delta t$. The term $Q_{i}\left(\vec{x}+\vec{c}_{i} \Delta t, t\right)$ represents the mass that is reflected from the surface and arrives in cell $\vec{x}+\vec{c}_{i} \Delta t$.

\section{Analytical solution of plane Poiseuille flow}

On the basis of the procedure described by He et al. [15], we will analyze the volumetric scheme as proposed by Chen et al. [12] in the case of plane Poiseuille flow. Consider a horizontal channel of height $L$, consisting of $m$ grid cells (see Fig. 1). The position of the lower and upper surfaces is such that the volume of all boundary cells is equal to $\alpha$, hence $L=(m-2)+2 \alpha$ (with $\Delta z=1)$. The facets of the surface are positioned between two vertical grid lines.

The flow is driven by a body force, i.e., $\vec{F}=\left(\rho G_{x}, 0,0\right)$, and periodic boundaries are imposed at both ends of the channel. For the analysis, we assume a steady-state flow, hence

$$
u_{z}=0, \frac{\partial u_{x}}{\partial x}=0, \frac{\partial u_{x}}{\partial t}=0
$$

This implies that
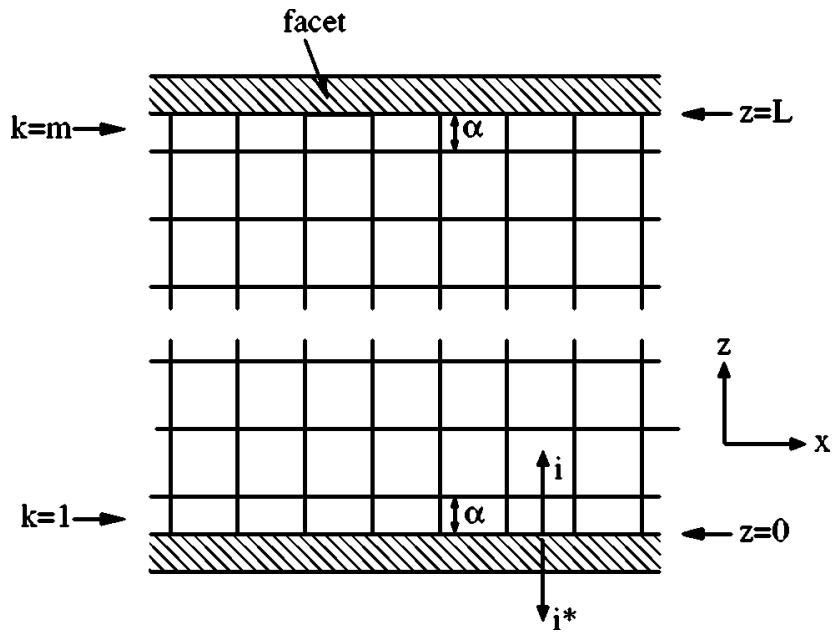

FIG. 1. Two-dimensional representation of a horizontal channel with height $L$ and noncubic boundary cells with volume $\alpha$ at the bottom of the channel.

$$
\frac{\partial N_{i}(\vec{x}, t)}{\partial x}=0, \quad \frac{\partial N_{i}(\vec{x}, t)}{\partial t}=0 .
$$

For the boundary cells on $z=z_{1}$ and $z=z_{2}$ (we only consider the cells at the bottom of the channel because of the symmetry of the flow field), the propagation step, described in the preceding section, evolves in the following manner. The incoming (and thus the reflected) mass per facet is

$$
\Gamma_{i *}^{i n, \alpha}=\Gamma_{i}^{\text {out }, \alpha}=N_{i *}\left(z_{1}, t_{*}\right)+(1-\alpha) N_{i *}\left(z_{2}, t_{*}\right) .
$$

According to Eq. (2), the reflected mass is redistributed uniformly in the cells at $z_{1}$ and $z_{2}$, hence the propagation step at these cells is given by

$$
\begin{gathered}
N_{i}\left(z_{1}, t+1\right)=\alpha\left[N_{i *}\left(z_{1}, t_{*}\right)+(1-\alpha) N_{i *}\left(z_{2}, t_{*}\right)\right], \\
N_{i *}\left(z_{1}, t+1\right)=\alpha N_{i *}\left(z_{2}, t_{*}\right), \\
N_{i}\left(z_{2}, t+1\right)=N_{i}\left(z_{1}, t_{*}\right)+(1-\alpha)\left[N_{i *}\left(z_{1}, t_{*}\right)\right. \\
\left.+(1-\alpha) N_{i *}\left(z_{2}, t_{*}\right)\right] \\
N_{i *}\left(z_{2}, t+1\right)=N_{i *}\left(z_{3}, t_{*}\right),
\end{gathered}
$$

where $t_{*}$ represents the moment after the collision step but before the propagation step, $i$ denotes all the directions from the surface, and $i^{*}$ denotes the directions to the surface. Note that this scheme can be used for any number of velocity directions. For the analysis, we use the two-dimensional nine-speed model on a square lattice and the BGK scheme (Fig. 2). The collision step then reads

$$
N_{i}\left(\vec{x}, t_{*}\right)=N_{i}(\vec{x}, t)-\frac{1}{\tau}\left[N_{i}(\vec{x}, t)-N_{i}^{e q}(\rho, \vec{u})\right]+t_{f, i} \Delta V \vec{c}_{i} \cdot \vec{F},
$$

where $\Delta V=\alpha$ for cells at $z=z_{1}$ and $\Delta V=1$ for cells at other positions. $\tau$ is the relaxation parameter, $t_{f, i}=0$ for $i=0$, 


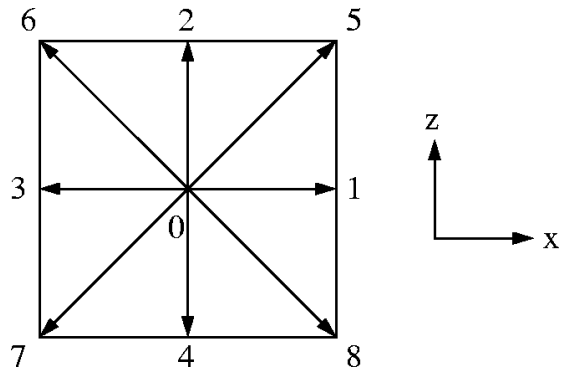

FIG. 2. Discrete velocity set for the two-dimensional nine-speed BGK scheme.

$t_{f, i}=\frac{1}{3}$ for $i=1,2,3,4$, and $t_{f, i}=\frac{1}{12}$ for $i=5,6,7,8$. The equilibrium distribution function $N_{i}^{e q}$ reads

$$
N_{i}^{e q}(\rho, \vec{u})=t_{p, i} \rho \Delta V\left(1+3\left(\vec{c}_{i} \cdot \vec{u}\right)+\frac{9}{2}\left(\vec{c}_{i} \cdot \vec{u}\right)^{2}-\frac{3}{2} \vec{u}^{2}\right),
$$

where $t_{p, i}=\frac{4}{9}$ for $i=0, t_{p, i}=\frac{1}{9}$ for $i=1,2,3,4$, and $t_{p, i}=\frac{1}{36}$ for $i=5,6,7,8$. The kinematic viscosity $\nu=(2 \tau-1) / 6$ and the speed of sound $c_{s}=\sqrt{\frac{1}{3}}$.

By using the definition of the velocity,

$$
\Delta V \rho u_{x}\left(z_{k}, t\right)=\sum_{i=0 \cdots b} c_{i, x} N_{i}\left(z_{k}, t\right)
$$

and Eqs. (6), (8), and (7), we can derive an equation with macroscopic quantities only (see the Appendix). This equation reads

$$
\mathcal{A}_{1} u_{x}\left(z_{1}\right)+\mathcal{A}_{2} u_{x}\left(z_{2}\right)+\mathcal{A}_{3} G_{x}=0
$$

with

$$
\begin{gathered}
\mathcal{A}_{1}=2 \tau+4 \alpha \tau-3 \alpha, \\
\mathcal{A}_{2}=\alpha-2 \alpha \tau, \\
\mathcal{A}_{3}=4 \alpha \tau-6 \alpha^{2}+6 \alpha^{2} \tau-4 \alpha \tau^{2}-4 \tau^{2} .
\end{gathered}
$$

A similar procedure is applied to grid cells at position $z_{2}, z_{3}$, etc. For position $z_{2}$, the following equation is obtained:

$$
\mathcal{B}_{1} u_{x}\left(z_{2}\right)+\mathcal{B}_{2} u_{x}\left(z_{3}\right)+\mathcal{B}_{3} G_{x}=0
$$

with

$$
\begin{aligned}
\mathcal{B}_{1}= & 17 \alpha \tau^{2}-50 \alpha \tau^{3}-19 \alpha^{3} \tau-8 \alpha^{3} \tau^{3}+22 \alpha^{3} \tau^{2}-56 \alpha^{2} \tau^{3} \\
& +32 \alpha \tau^{4}+16 \alpha^{2} \tau^{4}-6 \tau^{3}+12 \tau^{4}+5 \alpha^{3}+56 \alpha^{2} \tau^{2} \\
& -16 \alpha^{2} \tau,
\end{aligned}
$$

$$
\begin{aligned}
\mathcal{B}_{2}= & 8 \alpha^{2} \tau-16 \alpha^{2} \tau^{4}-16 \alpha \tau^{4}+40 \alpha^{2} \tau^{3}+13 \alpha^{3} \tau+8 \alpha^{3} \tau^{3} \\
& -18 \alpha^{3} \tau^{2}+2 \tau^{3}-4 \tau^{4}-3 \alpha^{3}+22 \alpha \tau^{3}-32 \alpha^{2} \tau^{2}-7 \alpha \tau^{2}, \\
\mathcal{B}_{3}= & -32 \alpha \tau^{5}-64 \alpha^{3} \tau+78 \alpha^{3} \tau^{2}-18 \alpha^{4} \tau-32 \alpha^{3} \tau^{3}+12 \alpha^{4} \tau^{2} \\
& +32 \alpha^{2} \tau^{4}+80 \alpha \tau^{4}-132 \alpha^{2} \tau^{3}+142 \alpha^{2} \tau^{2}-98 \alpha \tau^{3} \\
& -48 \alpha^{2} \tau+42 \alpha \tau^{2}-12 \tau^{3}+20 \tau^{4}-16 \tau^{5}+6 \alpha^{4}+18 \alpha^{3}
\end{aligned}
$$

For positions $z_{k=3 \cdots m-2}$, the equation reads

$$
\mathcal{C}_{1} u_{x}\left(z_{k-1}\right)-2 \mathcal{C}_{2} u_{x}\left(z_{k}\right)+\mathcal{C}_{3} u_{x}\left(z_{k+1}\right)+G=0
$$

with

$$
\mathcal{C}_{1}=\mathcal{C}_{2}=\mathcal{C}_{3}=\frac{2 \tau-1}{6}=\nu
$$

We clearly see that for the fluid nodes at $z_{k=3 \cdots m-2}$, a second-order central-difference scheme is obtained for the Navier-Stokes equations in the case of steady-state Poiseuille flow. Equations (10)-(12) are solved by substituting a velocity profile, i.e., a parabolic profile plus a numerical error $\Delta u_{x}$,

$$
u_{x}\left(z_{k}\right)=\frac{4 U_{\max }}{L^{2}} z_{k}\left(L-z_{k}\right)+\Delta u_{x}\left(z_{k}\right)
$$

with $U_{\max }=L^{2} G / 8 \nu$. By substituting the profile into Eq. (12), we obtain

$$
\Delta u_{x}\left(z_{k+1}\right)+\Delta u_{x}\left(z_{k-1}\right)-2 \Delta u_{x}\left(z_{k}\right)=0 .
$$

Due to the symmetry of the grid at $z=L / 2$, the simulated velocity profile in the channel (and thus the numerical error) is also symmetrical with respect to the center of the channel. This implies that Eq. (14) only holds when $\Delta u_{x}\left(z_{2}\right)$ $=\Delta u_{x}\left(z_{3}\right)=\cdots=\Delta u_{x}\left(z_{m-1}\right)$. The numerical error at position $z_{1}$ can, however, still be different from the error at the other positions, as confirmed by numerical simulations. Substituting the profile Eq. (13) into Eqs. (10) and (11) for $z_{1}$ $=\alpha-\frac{1}{2}, z_{2}=\alpha+\frac{1}{2}$, and $z_{3}=\alpha+1 \frac{1}{2}$ gives us two equations with two unknowns, because $\Delta u_{x}\left(z_{2}\right)=\Delta u_{x}\left(z_{3}\right)$. The numerical error reads

$$
\begin{gathered}
\Delta u_{x}\left(z_{1}\right)=\frac{1}{3} U_{\max } \frac{32 \tau^{3}-40 \alpha \tau^{2}-16 \tau^{2}+24 \alpha^{2} \tau-4 \alpha \tau+6 \tau-12 \alpha^{3}+12 \alpha^{2}-3 \alpha}{2 \tau-\alpha} \frac{1}{L^{2}}-4 U_{\max } \frac{\alpha \tau-\tau-\alpha^{2}+\alpha}{2 \tau-\alpha} \frac{1}{L}, \\
\Delta u_{x}\left(z_{k}\right)=\frac{1}{3} U_{\max } \frac{32 \tau^{3}-16 \alpha \tau^{2}-40 \tau^{2}+20 \alpha \tau+6 \tau-12 \alpha^{3}+12 \alpha^{2}-3 \alpha}{2 \tau-\alpha} \frac{1}{L^{2}}+4 U_{\max } \frac{\alpha(\alpha-1)}{2 \tau-\alpha} \frac{1}{L} .
\end{gathered}
$$




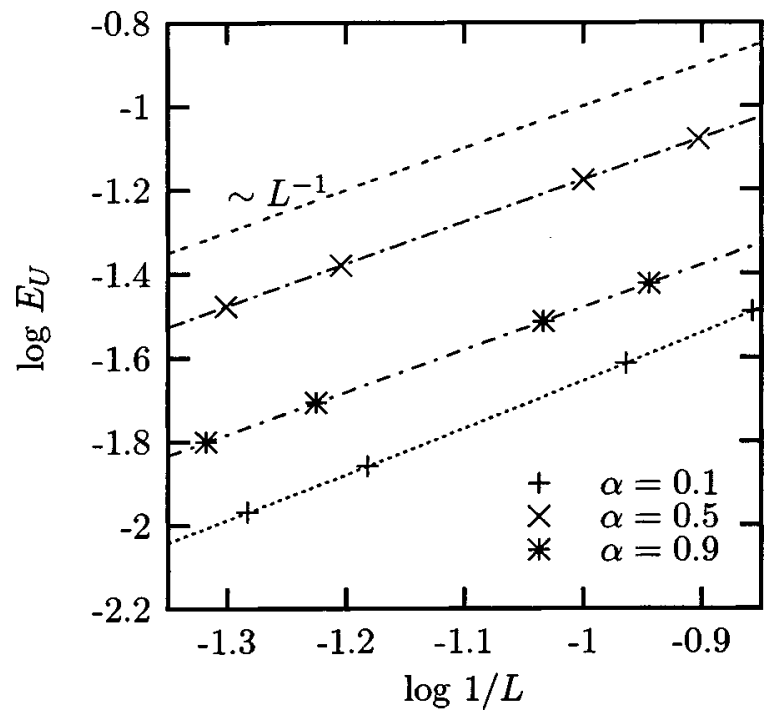

FIG. 3. The relative error in the velocity at the centerline of a channel against grid spacing. The size of the channel was varied in the range from $L=8$ to $L=20, \tau$ was set to 1 . The volume of the boundary cells at the bottom and top of the channel was varied in the range $\alpha=0.1-0.9$. The points correspond to the simulation data, the lines represent the analytical findings.

From Eq. (15) it is evident that the method is first-order accurate with respect to the grid spacing $1 / L$ [the error is of the form $\left.\Delta u_{x}(z)=E_{1} / L+E_{2} / L^{2}\right]$. Simulations confirm this result, as shown in Fig. 3.

Note that the accuracy is second order in the case of $\alpha$ $=1$, which is equivalent to the common bounce-back scheme with walls placed halfway between two grid nodes (known as halfway Bounce-Back method). The error then reads

$$
\Delta u_{x}\left(z_{k}\right)=\frac{U_{\max }\left(16 \tau^{2}-20 \tau+3\right)}{3 L^{2}}
$$

for all positions in the channel. This result was also found by He et al. [15].

In summary, the main result obtained so far is that the volumetric scheme studied in this section is first-order accurate with respect to the lattice spacing. One important assumption in this scheme is that the reflected mass is distributed uniformly in the boundary cells. This may induce numerical diffusion and affect the accuracy of the scheme.

\section{B. Schemes by Bouzidi et al. and Verberg and Ladd}

Several authors used interpolation of particle densities in the vicinity of the surface to improve the accuracy of their method. The resulting density profile is then reflected (i.e., bounced back) into the fluid. This was recently done by Verberg and Ladd [11] and by Bouzidi et al. [13]. Verberg and Ladd used a volumetric approach with refined staircase shaped surfaces, while the method of Bouzidi et al. is based on densities located at grid nodes.

To illustrate the effect of using interpolation of the densities on the absolute error in the velocity, the analysis of the preceding section is also applied to the method of Bouzidi et al. Suppose that the distance between the grid nodes at $z_{1}$

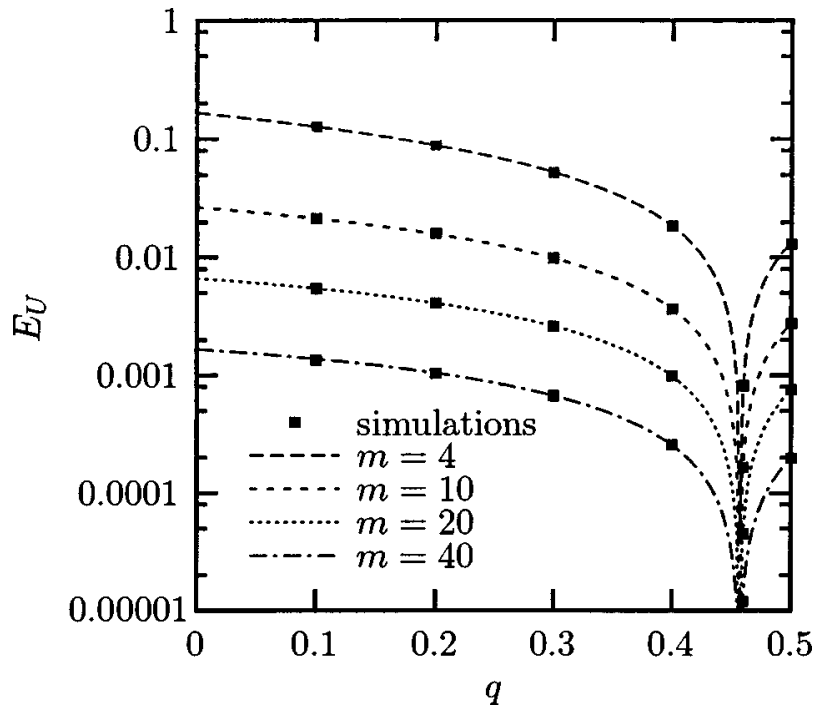

FIG. 4. The relative error in the velocity at the centerline of a channel as a function of $q$ in the case of the first-order interpolation scheme as proposed by Bouzidi et al. The size of the channel was varied in the range of $m=4$ to $m=40(L=m-1+2 q), \tau$ was set to 1 . The points correspond to the simulation data, the lines to the analytically found numerical error of Eq. (18).

and the wall is denoted by $q$ (in the volumetric approach, $\alpha=q+1 / 2$ for $q<1 / 2$ and $\alpha=q-1 / 2$ for $q \geqslant 1 / 2)$. The propagation step in the case of a first-order interpolation of the densities reads

$$
\begin{aligned}
& n_{i}\left(z_{1}, t+1\right)=2 q n_{i *}\left(z_{1}, t_{*}\right)+(1-2 q) n_{i *}\left(z_{2}, t_{*}\right) \quad\left(q<\frac{1}{2}\right) \\
& n_{i}\left(z_{1}, t+1\right)=\frac{1}{2 q} n_{i *}\left(z_{1}, t_{*}\right)+\frac{2 q-1}{2 q} n_{i}\left(z_{1}, t_{*}\right) \quad\left(q \geqslant \frac{1}{2}\right) .
\end{aligned}
$$

The two different expressions in Eq. (17) are necessary, because of stability reasons [13]. By applying the same analysis as described in the preceding section, we obtain for the error in the velocity at $z_{1 \cdots m}$ in the case of $q<1 / 2$,

$$
\Delta u_{x}\left(z_{1 \cdots m}\right)=\frac{U_{\max }\left(16 \tau^{2}-8 \tau-24 q \tau+12 q-12 q^{2}\right)}{3 L^{2}},
$$

which indeed indicates second-order accuracy. Simulations confirm Eq. (18) (see Fig. 4).

One may also use a second-order interpolation based on the densities on three neighboring nodes, $z_{1}, z_{2}$, and $z_{3}$ [13]. The propagation step then reads

$$
\begin{aligned}
n_{i}\left(z_{1}, t+1\right)= & q(2 q+1) n_{i *}\left(z_{1}, t_{*}\right) \\
& +(1+2 q)(1-2 q) n_{i *}\left(z_{2}, t_{*}\right) \\
& -q(1-2 q) n_{i *}\left(z_{3}, t_{*}\right) \quad\left(q<\frac{1}{2}\right)
\end{aligned}
$$




$$
\begin{aligned}
n_{i}\left(z_{1}, t+1\right)= & \frac{1}{q(2 q+1)} n_{i *}\left(z_{1}, t_{*}\right)+\frac{2 q-1}{q} n_{i}\left(z_{1}, t_{*}\right) \\
& +\frac{1-2 q}{1+2 q} n_{i}\left(z_{2}, t_{*}\right) \quad\left(q \geqslant \frac{1}{2}\right)
\end{aligned}
$$

After the error analysis, the error for the second-order interpolation for $q<1 / 2$ reads

$$
\Delta u_{x}\left(z_{1 \cdots m}\right)=\frac{U_{\max }\left(16 \tau^{2}-8 \tau-24 q \tau+12 q^{2}\right)}{3 L^{2}} .
$$

As expected, we find that the absolute error is smaller in the case of second-order interpolation. Note that both errors (related to first-order and second-order interpolations) reduce to the error for the halfway bounce back for $q=1 / 2$ ( $\alpha$ $=1)$. Despite the simplicity of this scheme, it seems to have a drawback. The scheme does not conserve mass, because of errors associated with interpolation of densities residing on grid nodes.

Verberg and Ladd also analyzed the numerical error of the volumetric method presented in Ref. [11]. The error in the case of plane Poiseuille flow for the nine-speed BGK scheme at $z_{2} \cdots m-1$ reads

$$
\begin{aligned}
\Delta u_{x}\left(z_{2 \cdots m-1}\right)= & \frac{U_{\max }\left(16 \tau^{2}-16 \tau+1+6 \alpha^{2}-6 \alpha\right)}{3 L^{2}} \\
& +\frac{U_{\max } \alpha^{2}(\alpha-1)^{2}}{L^{4}},
\end{aligned}
$$

which also shows second-order accuracy with respect to grid spacing ( $\alpha$ denotes the volume of a cell at the boundary). However, the accuracy is reduced to first order in the case of more complex geometries, such as a skew channel with respect to the underlying grid [11].

\section{MODIFIED VOLUMETRIC SCHEMES}

\section{A. Chen's scheme with interpolation}

We now return to the volumetric method of Chen et al. [12], which was found to be first-order accurate in space in the case of plane Poiseuille flow. This behavior may be attributed to the uniform redistribution of the reflected mass across the boundary cells. In fact, the uniformly reflected mass profile can be regarded as a zeroth-order approximation of the actual profile. In the preceding section, we have seen that, by applying a higher-order interpolation, a second-order accuracy with respect to the discretization of space can be achieved. We shall therefore apply these ideas to the volumetric scheme of Chen et al.

Consider a facet with surface $A^{\alpha}$ and the parallelepiped with volume $\Phi_{i}^{\alpha}$, intersecting some grid cells in the vicinity of the surface [see Fig. 5(a)]. Some of these grid cells are directly cut by the facet (type-I cells), others are not (type-II cells). The intersecting volumes of the parallelepiped and cells of the same type are summed and rearranged, such that two new volumes are created [see Fig. 5(b)]. The idea now is

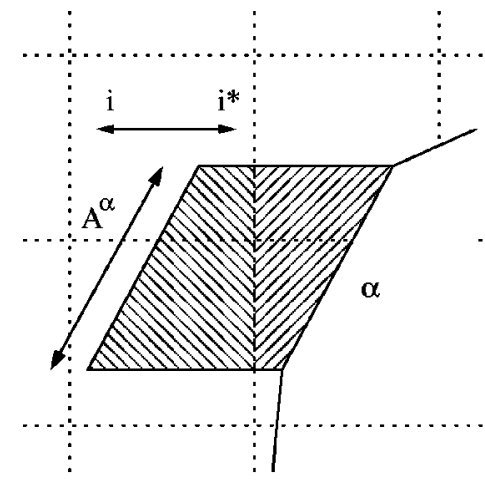

a

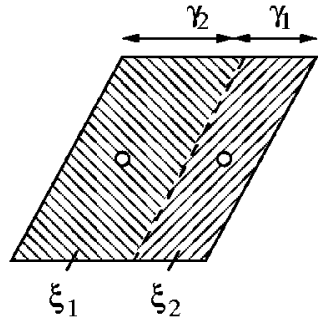

b
FIG. 5. Two-dimensional representation of the intersecting volumes of type I $\left(\mathcal{V}_{\mathrm{I}}\right.$, indicated with $\left./ / /\right)$ and type II $\left(\mathcal{V}_{\mathrm{II}}\right.$, indicated with $\backslash \backslash \backslash$ ) cells in a parallelepiped, extruded from a facet with area $A^{\alpha}$.

that the density profile of the incoming mass is approximated by interpolating the densities between the centers of these two volumes (i.e., $\xi_{1}$ and $\xi_{2}$ ). Subsequently, this profile is reflected (i.e., bounced back) by facet $\alpha$, resulting in a profile of reflected densities.

The lengths of the two new volumes are

$$
\begin{aligned}
& \gamma_{1}=\mathcal{V}_{\mathrm{I}} / A_{*}^{\alpha}=\sum_{\vec{x} \in \text { type-I cells }} V_{i}^{\alpha}(\vec{x}) / A_{*}^{\alpha}, \\
& \gamma_{2}=\mathcal{V}_{\mathrm{II}} / A_{*}^{\alpha}=\sum_{\vec{x} \in \text { type-II cells }} V_{i}^{\alpha}(\vec{x}) / A_{*}^{\alpha},
\end{aligned}
$$

where $\mathcal{V}_{\mathrm{I}}$ and $\mathcal{V}_{\mathrm{II}}$ represent the volumes, $\gamma_{1}+\gamma_{2}=\left|\vec{c}_{i}\right|$ and $A_{*}^{\alpha}=\left(\vec{c}_{i} \cdot \vec{n}_{\alpha}\right) /\left|\vec{c}_{i}\right| A^{\alpha}$. The densities in the new volumes can be approximated by averaging the densities of the boundary cells involved, according to

$$
\begin{aligned}
& n_{i}\left(\xi_{1}\right)=\frac{1}{\mathcal{V}_{\mathrm{I}}} \sum_{\vec{x} \in \text { type-I cells }} V_{i}^{\alpha}(\vec{x}) n_{i}(\vec{x}), \\
& n_{i}\left(\xi_{2}\right)=\frac{1}{\mathcal{V}_{\mathrm{II}}} \sum_{\vec{x} \in \text { type-II cells }} V_{i}^{\alpha}(\vec{x}) n_{i}(\vec{x}) .
\end{aligned}
$$

Now that the volumes and densities have been simplified, the densities moving towards the surface (i.e., the $i^{*}$ direction) can easily be interpolated according to

$$
n_{i *}(\xi)=2 \frac{\left[n_{i *}\left(\xi_{2}\right)-n_{i *}\left(\xi_{1}\right)\right]}{\gamma_{1}+\gamma_{2}} \xi+n_{i *}\left(\xi_{1}\right),
$$

where $\xi_{1}$ is set to the origin of the $\xi$ axis. The total incoming mass is obtained by integrating the interpolated densities over the total volume of the parallelepiped

$$
\begin{aligned}
\Gamma_{i *}^{i n, \alpha} & =A_{*}^{\alpha} \int_{-(1 / 2) \gamma_{1}}^{(1 / 2) \gamma_{1}+\gamma_{2}} n_{i *}(\xi) d \xi \\
& =A_{*}^{\alpha}\left[\gamma_{1} n_{i *}\left(\xi_{1}\right)+\gamma_{2} n_{i *}\left(\xi_{2}\right)\right],
\end{aligned}
$$

which is the same amount of mass as in Eq. (5). 
The interpolated densities are reflected (i.e., bounced back) by facet $\alpha$. The resulting density profile then reads

$$
n_{i}(\xi)=-2 \frac{\left[n_{i *}\left(\xi_{2}\right)-n_{i *}\left(\xi_{1}\right)\right]}{\gamma_{1}+\gamma_{2}}\left(\xi-\gamma_{2}\right)+n_{i *}\left(\xi_{1}\right) .
$$

By applying this reflection, a more accurate redistribution of the reflected mass is obtained compared to the method described in Sec. II A. The redistribution in the volumes with center points $\xi_{1}$ and $\xi_{2}$ results from the integration of the reflected density profile over these volumes,

$$
\begin{aligned}
\Gamma_{i}^{\text {out }, \alpha}\left(\xi_{1}\right) & =A_{*}^{\alpha} \int_{-(1 / 2) \gamma_{1}}^{(1 / 2) \gamma_{1}} n_{i}(\xi) d \xi \\
& =A_{*}^{\alpha}\left[\frac{2 \gamma_{1} \gamma_{2}}{\gamma_{1}+\gamma_{2}} n_{i *}\left(\xi_{2}\right)+\frac{\left(\gamma_{1}-\gamma_{2}\right) \gamma_{1}}{\gamma_{1}+\gamma_{2}} n_{i *}\left(\xi_{1}\right)\right], \\
\Gamma_{i}^{\text {out } \alpha}\left(\xi_{2}\right) & =A_{*}^{\alpha} \int_{(1 / 2) \gamma_{1}}^{(1 / 2) \gamma_{1}+\gamma_{2}} n_{i}(\xi) d \xi \\
& =A_{*}^{\alpha}\left[\frac{\left(\gamma_{2}-\gamma_{1}\right) \gamma_{2}}{\gamma_{1}+\gamma_{2}} n_{i *}\left(\xi_{2}\right)+\frac{2 \gamma_{1} \gamma_{2}}{\gamma_{1}+\gamma_{2}} n_{i *}\left(\xi_{1}\right)\right] .
\end{aligned}
$$

Here, the difference between the method of Sec. II A and the interpolation method can clearly be seen; the reflected mass is now redistributed on two positions in space $\left(\xi_{1}\right.$ and $\left.\xi_{2}\right)$ instead of one. Note that mass is conserved by definition $\left[\Gamma_{i *}^{i n, \alpha}=\Gamma_{i}^{\text {out }, \alpha}\left(\xi_{1}\right)+\Gamma_{i}^{\text {out }, \alpha}\left(\xi_{2}\right)\right]$.

It should be realized that the method as described above is not the only way to approximate the density profile in the parallelepiped. Another route is to split the parallelepiped into two equal volumes $\left(\gamma_{1}=\gamma_{2}\right)$ and calculate the geometrical intersection with the grid cells. In this way, the exact amount of mass present in these volumes can be determined. A disadvantage of this method is that it requires additional computational effort for the calculation of the intersections. For this reason, we restrict the following analysis to the more simple method described at the beginning of this section.

\section{Analytical solution of plane Poiseuille flow}

The same analysis regarding the accuracy of the method with respect to the grid spacing can be performed as described in Sec. II A. The numerical error is found to be

$$
\begin{aligned}
& \Delta u_{x}\left(z_{1}\right)=\frac{1}{3} U_{\max } \frac{16 \tau^{2}-24 \tau \alpha+4 \tau+12 \alpha^{2}-12 \alpha+3}{L^{2}}, \\
& \Delta u_{x}\left(z_{k=2 \cdots m-1}\right)=\frac{1}{3} U_{\max } \frac{16 \tau^{2}-20 \tau+12 \alpha^{2}-12 \alpha+3}{L^{2}} .
\end{aligned}
$$

Hence, the error in the flow field shows a second-order dependency on the grid spacing $\left(\sim 1 / L^{2}\right)$, as is also evident from Fig. 6. Moreover, from Eq. (28) it follows that the error at $z_{k=2 \cdots m-1}$ has a minimum at $\alpha=0.5$ and a maximum at

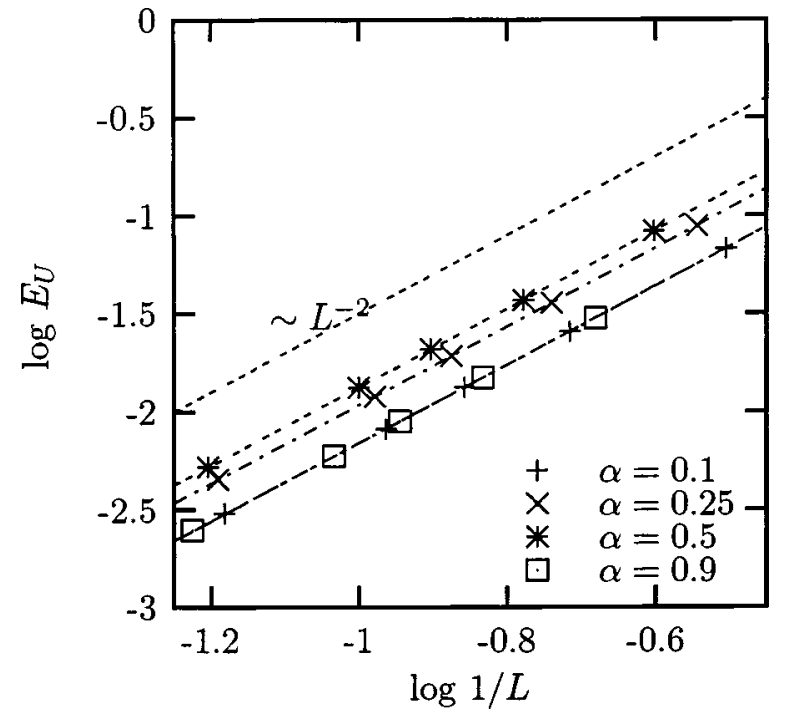

FIG. 6. The relative error in the velocity at the centerline of a channel against the grid spacing. The size of the channel was varied in the range of $L=4-16, \tau$ was set to 1 . The volume of the boundary cells at the bottom and top of the channel was varied in the range of $\alpha=0.1-0.9$. The points correspond to the simulation data, the lines represent the analytical findings of Eq. (28).

$\alpha=0,1$ for a fixed value of $\tau$. Note that for $\alpha=q+1 / 2$ and $q<1 / 2$, Eq. (28) is equivalent to the expression obtained for the method by Bouzidi et al. with second-order interpolation [Eq. (20)].

By rearranging the error at $z_{k=2 \cdots m-1}$, we obtain

$$
\begin{aligned}
\Delta u_{x}\left(z_{k=2 \cdots m-1}\right)= & \frac{U_{\max }\left(16 \tau^{2}-20 \tau+3\right)}{3 L^{2}} \\
& +\frac{4 U_{\max } \alpha(\alpha-1)}{L^{2}} .
\end{aligned}
$$

Here, we identify the error of the halfway bounce-back method [Eq. (16)] plus a contribution solely due to the fractional volume of the boundary cells. In the case of $\alpha=1$, the original halfway bounce-back method is recovered as expected (this also applies to the error at $z_{1}$ ).

\section{Analysis for a skew channel}

An important question is whether the interpolation procedure described above will also lead to higher-order accuracy for more complex cases. We, therefore, performed simulations on plane Poiseuille flow in a skew channel, where the boundary is positioned at a nonzero angle with respect to the underlying grid. The results are shown in Fig. 7. Data obtained for the original scheme of Chen et al. are also included in the graph. It is clear that, although linear interpolation reduces the error in the velocity, the accuracy remains first order. These findings are similar to the results of Verberg and Ladd [11], where the accuracy degenerates from second order to first order as the angle deviates from zero. Moreover, other interpolation schemes for approximating the density profile near the boundary (such as the technique sug- 


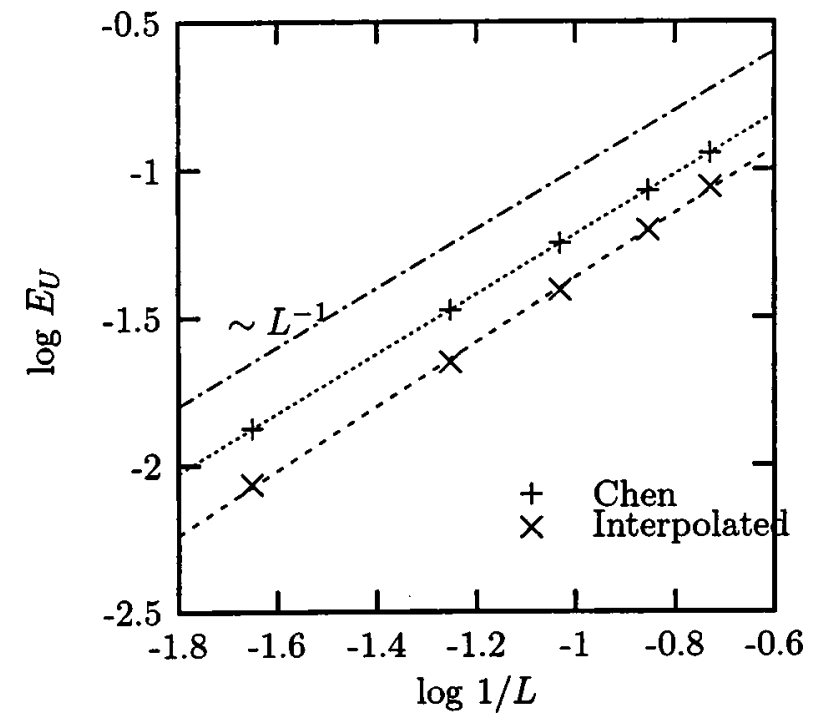

FIG. 7. The relative error in the centerline velocity of a skew channel against the grid spacing. The relaxation parameter $\tau$ was set to 1 . Simulations were performed for an angle $\phi=\arctan (0.5)$ with respect to the grid. Results are shown for Chen's method with and without application of an interpolation scheme.

gested at the end of Sec. III A) or increasing the number of facets, did not result in higher-order accuracy.

We believe that, even though the particle distributions are interpolated near the wall, first-order accuracy is still obtained due to the way the volumes I and II in Fig. 5 are lumped. Moreover, $\mathcal{V}_{\mathrm{I}}$ and $\mathcal{V}_{\mathrm{II}}$ are determined exactly, but the geometrical structure of the boundary cells is not incorporated. Consequently, the shape of the surface is not captured accurately enough to obtain second-order accuracy.

\section{B. A different volumetric approach}

In the preceding section, it was suggested that volumes of boundary cells only do not describe the surface accurately enough, therefore resulting in a first-order bounce-back scheme. In order to incorporate the shape of these cells, additional geometrical information of the boundary is needed. One way to accomplish this is by introducing a weighing factor $\alpha_{i}$ in each direction $i$ separately, instead of a single volume (see Fig. 8). This weighing factor is determined by the intersection of a specific link and the corresponding boundary cell, which can be considered as an effective volume along each link. In the case of a cubic boundary cell, $\alpha_{i}$ is equal to 1 for all $i$. For noncubic boundary cells, $\alpha_{i}$ may vary in the range of $0.5 \leqslant \alpha_{i} \leqslant 1.5$. The weighing factors for opposite directions are the same, thus $\alpha_{i}=\alpha_{i *}$. E.g., for cell $\left(x_{1}, z_{1}\right)$ in Fig. 8, these are $\alpha_{1}=\alpha_{3}=1, \alpha_{2}=\alpha_{4}=\frac{7}{8}, \alpha_{5}$ $=\alpha_{7}=1$, and $\alpha_{6}=\alpha_{8}=\frac{4}{5}$. With the definition of $\alpha_{i}$, we can now define the particle density that moves in direction $i$, which is $n_{i} \equiv N_{i} / \alpha_{i}$ (instead of $\left.N_{i} / \Delta V\right)$. This definition enables us to set up a mass conservative bounce-back scheme.

The propagation steps (in terms of densities) on the shifted grid nodes $\left(x_{2}, z_{1}^{\prime}\right)$ and $\left(x_{2}, z_{2}^{\prime}\right)$ in directions $i=2$ and $i *=4 \mathrm{read}$

$$
n_{i}\left(z_{1}^{\prime}, t+1\right)=n_{i *}\left(z_{1}^{\prime}, t_{*}\right),
$$

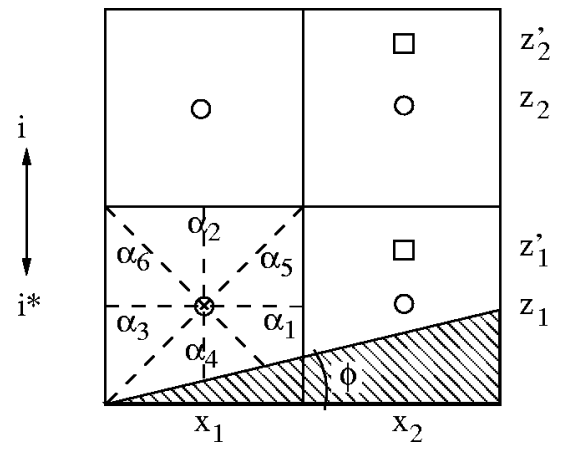

FIG. 8. Example of the volumes $\alpha_{i}$ in each direction $i$ near a boundary. The circles represent the centers of the grid cells [with coordinates $(x, z)]$ and the squares represent the shifted centers in direction $i$ [with coordinates $\left.\left(x, z^{\prime}\right)\right]$. Here, the surface has an angle $\phi=\arctan (1 / 4)$ and is located at position $\left(x_{1}-\frac{1}{2}\left|\vec{c}_{i}\right|, z_{1}-\frac{1}{2}\left|\vec{c}_{i}\right|\right)$.

$$
\begin{gathered}
n_{i *}\left(z_{1}^{\prime}, t+1\right)=n_{i *}\left(z_{2}^{\prime}, t_{*}\right), \\
n_{i}\left(z_{2}^{\prime}, t+1\right)=n_{i}\left(z_{1}^{\prime}, t_{*}\right), \\
n_{i *}\left(z_{2}^{\prime}, t+1\right)=n_{i *}\left(z_{3}^{\prime}, t_{*}\right),
\end{gathered}
$$

where the grid nodes $z_{k}^{\prime}$ are shifted such that the distance from the boundary to the first grid node $z_{1}^{\prime}$ equals $1 / 2$ (the $x_{2}$ coordinate is omitted for clarity). Hence, Eqs. (30) represent a normal bounce-back scheme for a wall positioned halfway between two grid nodes. We can estimate the density in directions $i$ and $i^{*}$ on $z_{k}^{\prime}$ by linearly interpolating the densities on $\left(x_{2}, z_{k}\right)$ and $\left(x_{2}, z_{k+1}\right)$ according to

$$
n_{i}\left(z_{k}^{\prime}, t\right)=\alpha_{i} n_{i}\left(z_{k}, t\right)+\left(1-\alpha_{i}\right) n_{i}\left(z_{k+1}, t\right) .
$$

By substituting Eq. (31) in Eq. (30), we obtain a set of equations in terms of densities on the grid nodes $(x, z)$,

$$
\begin{array}{r}
k=1, \quad \alpha_{i} n_{i}\left(z_{1}, t+1\right)+\left(1-\alpha_{i}\right) n_{i}\left(z_{2}, t+1\right) \\
=\alpha_{i} n_{i *}\left(z_{1}, t_{*}\right)+\left(1-\alpha_{i}\right) n_{i *}\left(z_{2}, t_{*}\right), \\
\alpha_{i} n_{i *}\left(z_{1}, t+1\right)+\left(1-\alpha_{i}\right) N_{i *}\left(z_{2}, t+1\right) \\
=\alpha_{i} n_{i *}\left(z_{2}, t_{*}\right)+\left(1-\alpha_{i}\right) n_{i *}\left(z_{3}, t_{*}\right), \\
k>1, \quad \alpha_{i} n_{i}\left(z_{k}, t+1\right)+\left(1-\alpha_{i}\right) n_{i}\left(z_{k+1}, t+1\right) \\
=\alpha_{i} n_{i}\left(z_{k-1}, t_{*}\right)+\left(1-\alpha_{i}\right) n_{i}\left(z_{k}, t_{*}\right), \\
\alpha_{i} n_{i *}\left(z_{k}, t+1\right)+\left(1-\alpha_{i}\right) n_{i *}\left(z_{k+1}, t+1\right) \\
=\alpha_{i} n_{i *}\left(z_{k+1}, t_{*}\right)+\left(1-\alpha_{i}\right) n_{i *}\left(z_{k+2}, t_{*}\right) .
\end{array}
$$

Because, in the bulk of the fluid (i.e., for $k>1$ ), $n_{i}\left(z_{k+1}, t+1\right)=n_{i}\left(z_{k}, t_{*}\right) \quad$ and $\quad n_{i *}\left(z_{k}, t+1\right)$ $=n_{i *}\left(z_{k+1}, t_{*}\right)$, we may simplify Eq. (32) as follows:

$$
\begin{aligned}
k=1, n_{i}\left(z_{1}, t+1\right)= & \left(1-\frac{1}{\alpha_{i}}\right) n_{i}\left(z_{1}, t_{*}\right)+n_{i *}\left(z_{1}, t_{*}\right) \\
& +\left(\frac{1}{\alpha_{i}}-1\right) n_{i *}\left(z_{2}, t_{*}\right),
\end{aligned}
$$




$$
\begin{gathered}
n_{i *}\left(z_{1}, t+1\right)=n_{i *}\left(z_{2}, t_{*}\right), \\
k>1, \quad n_{i}\left(z_{k+1}, t+1\right)=n_{i}\left(z_{k}, t_{*}\right), \\
n_{i *}\left(z_{k+1}, t+1\right)=n_{i *}\left(z_{k+2}, t_{*}\right) .
\end{gathered}
$$

By using Eqs. (33), it can be shown that (i) the bounceback scheme is mass conservative $\left[\sum_{i} N_{i}(\vec{x}, t+1)\right.$ $\left.=\sum_{i} N_{i}\left(\vec{x}, t_{*}\right)\right]$ (ii) all densities propagate to the adjacent grid node in the usual way, except (of course) for the reflected density $n_{i}\left(z_{1}, t+1\right)$.

The collision step, $n_{i}\left(x, t_{*}\right)=(1-1 / \tau) n_{i}(x, t)$ $+(1 / \tau) n_{i}^{e q}(x, t)$ can be rewritten in terms of mass, and is given by $N_{i}\left(t_{*}\right)=(1-1 / \tau) N_{i}(t)+(1 / \tau) \alpha_{i} n_{i}^{e q}(t)$. It follows that (i) in the collision step, mass density and momentum density are conserved quantities, i.e., $\quad \sum_{i} n_{i}\left(\vec{x}, t_{*}\right)$ $=\sum_{i} n_{i}(\vec{x}, t)$ and $\sum_{i} c_{i} n_{i}\left(\vec{x}, t_{*}\right)=\sum_{i} c_{i} n_{i}(\vec{x}, t)$, respectively. (ii) the total mass is not conserved, $\sum_{i} N_{i}\left(\vec{x}, t_{*}\right) \equiv \sum_{i} \alpha_{i} n_{i}^{e q}(\vec{x}, t)$ $\neq \sum_{i} N_{i}(\vec{x}, t)$. After some simple algebraic manipulations, it is found that the deviation in the total mass is of the order $\sim|\vec{u}|^{2}$. For Stokes flow, these quadratic terms in the equilibrium distribution are not needed, and then this scheme does conserve mass.

\section{Analytical solution for nonskew plane Poiseuille flow}

Following the method described in Sec. II A, the error in the velocity is given by

$$
\begin{aligned}
\Delta u_{x}\left(z_{k=2 \cdots m-1}\right)= & \frac{U_{\max }\left(16 \tau^{2}-20 \tau+3\right)}{3 L^{2}} \\
& +\frac{4 U_{\max } \alpha_{i}\left(1-\alpha_{i}\right)}{L^{2}}
\end{aligned}
$$

which indicates that the method is second-order accurate for this specific situation. Note that all weighing factors $\alpha_{i}$ for the directions toward the wall are then equal.

\section{Analysis for a skew channel}

In the case of a skew channel, the position of the boundary with respect to the grid and the angle of the channel were varied. The results can be found in Fig. 9. It is clear that the method is second-order accurate in all cases. The highest deviation in the total mass with respect to the total mass in the channel, indicated with $\Delta \mu / N_{t} 3 L_{c} /\left(2 U_{\max }\right) 1 / \mu=-4$ $\times 10^{-4}$ (with $\Delta \mu$ being the deviation in the total mass $\mu, N_{t}$ being the number of time steps related to the mass deviation, and $L_{c}$ being the length of the channel) is found for the smallest channel, because the velocities near the boundary are then relatively high. It is also found that $\Delta \mu$ is smaller than in the scheme of Bouzidi et al. Note that the leakage of mass can be corrected for in an ad hoc way, by simply redistributing $\Delta \mu$ over all boundary cells according to $\Delta N_{i}$ $=\Delta \mu \alpha_{i} / \Sigma_{i} \alpha_{i}$. This slightly reduces the accuracy of the method (slope $\sim 1.8$ ), but results, in the case of a skew channel, in a satisfactory flow profile. We are aware, however,

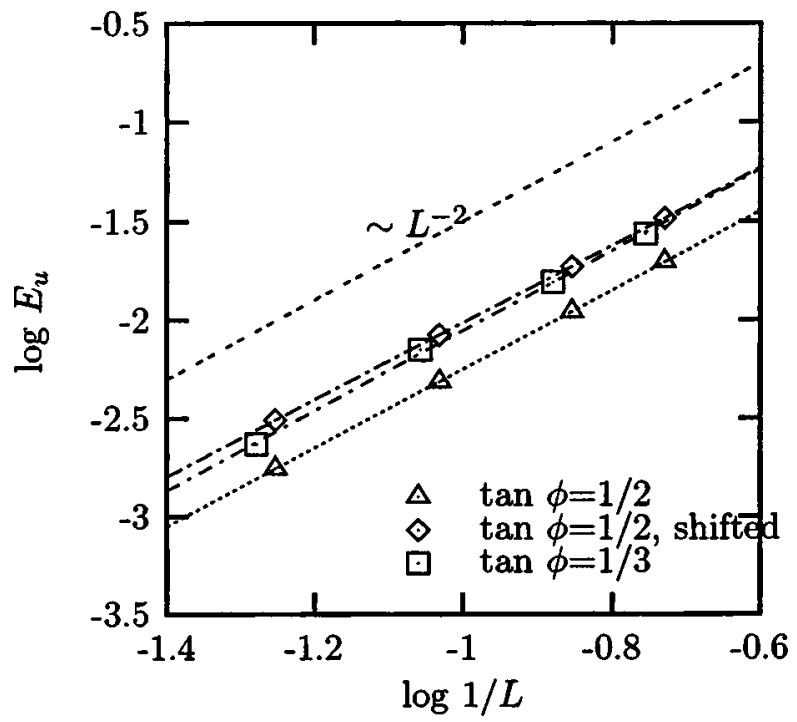

FIG. 9. The relative error in the centerline velocity in a skew channel against the grid spacing. The vertical size of the channel was varied in the range of $L=6-20$. The relaxation parameter $\tau$ was set to 1 . For $\tan \phi=1 / 2$, two positions with respect to the grid were taken [origins at $\left(\frac{1}{2}, \frac{1}{2}\right)(\triangle)$ and $\left(\frac{1}{2}, \frac{3}{4}\right)(\diamond)$ for the lower wall]. For $\tan \phi=1 / 3$, the origin was located at $\left(\frac{3}{2}, 1\right)(\square)$. The upper wall was shifted according to the channel height.

that this correction is not a fundamental solution to this problem. Such a solution may require different collision rules, which are not restricted to the discrete velocity set $c_{i}$ employed here. Some details about this research topic can be found in Refs. [16,17].

\section{CONCLUSION}

In this paper, several boundary techniques are analyzed for imposing no-slip walls in lattice-Boltzmann schemes. More specifically, the accuracy in the velocity field with respect to the spatial discretization is studied analytically and numerically. First, the volumetric boundary method as proposed by Chen et al. [12] is considered. An analytical expression for the error in the velocity in the case of plane Poiseuille flow is derived, pursuing the approach due to $\mathrm{He}$ et al. [15]. It is found that the method is first-order accurate in space. Furthermore, the analysis shows that the error depends on the relaxation parameter and the volume of the boundary cells.

A similar study elucidates that the boundary method proposed by Bouzidi et al. [13] is second-order accurate in space. This method uses linear or quadratic interpolation of the particle densities close to the wall, in contrast to the uniform redistribution of the reflected mass inherent to the method of Chen et al. The scheme of Bouzidi et al., however, does not conserve mass. A linear approximation of the reflected density profile is therefore applied to the volumetric scheme of Chen et al. For this modified scheme, it is shown analytically that, in the case of plane Poiseuille flow with boundaries aligned parallel to the underlying grid, secondorder accuracy in space is obtained. For boundaries posi- 
tioned at an angle with respect to the underlying grid, however, this scheme is first-order accurate only. This may be due to the fact that volumes only do not provide an adequate description of the shape of the boundary.

Therefore, these volumetric ideas are extended to a scheme where the boundary is resolved more accurately. A weighing factor is introduced in each direction $i$, representing an effective volume along each link. This indeed results in second-order accuracy in space. The mass is conserved in the propagation step, but still not in the collision step. The deviation in the total mass, however, is very small and proportional to the second-order terms in the equilibrium distribution. Consequently, the scheme is a priori mass conservative for Stokes flow. Though only flat surfaces are studied in this paper, the method can be applied to any boundary. In some specific cases, such as a grid node located close to an inner corner of a concave surface, the scheme needs to be modified because of the lack of the second grid node at $z_{2}$.

The extra computational time, introduced by the proposed scheme, is found to be very small when the weighing factors are calculated and stored beforehand. The computational time for the calculation of the weighing factors is about $5 \mathrm{~s}$ per 1000 boundary grid nodes on a $1.8 \mathrm{GHz} \mathrm{PC}$ in the case of a 15-speed lattice-Boltzmann scheme, which is relatively very small compared to the overall computation time. The weighing factors need an additional amount of memory, which is proportional to the number of boundary nodes, the number of discrete velocity directions, and the number of bytes used per floating point number. In most cases, the number of boundary nodes is much smaller than the total amount of grid nodes, hence the additional memory required is small.

Formulation of a method that does conserve mass and is second-order accurate in space in all cases is an important subject for future work. Moreover, this study will be extended to more complex flows and geometries, such as turbulent channel flow and flow around curved moving boundaries, following the method as proposed by Rohde et al. [18]. Ultimately, the aim is to apply these advanced boundary techniques to real-life applications such as mixing processes in a stirred vessel.

\section{APPENDIX: DERIVATION OF EQ. (10)}

The lattice-Boltzmann equation for directions $i$ $=1,3,5,6,7,8$ for the cells at $z_{1}$ and $z_{2}$, together with the simplifications for Poiseuille flow in Eq. (4) and the reflection rules of Eqs. (6), read

$$
\begin{aligned}
N_{1}\left(z_{1}\right)= & \frac{1}{9} \rho \alpha\left[1+3 u_{x}\left(z_{1}\right)+3 u_{x}\left(z_{1}\right)^{2}\right]+\frac{1}{3} \rho \alpha \tau G_{x}, \\
N_{3}\left(z_{1}\right)= & \frac{1}{9} \rho \alpha\left[1-3 u_{x}\left(z_{1}\right)+3 u_{x}\left(z_{1}\right)^{2}\right]-\frac{1}{3} \rho \alpha \tau G_{x}, \\
N_{5}\left(z_{1}\right)= & \alpha\left(N_{7}\left(z_{1}\right)-1 / \tau\left\{N_{7}\left(z_{1}\right)-\frac{1}{36} \rho \alpha\left[1-3 u_{x}\left(z_{1}\right)\right.\right.\right. \\
& \left.\left.+3 u_{x}\left(z_{1}\right)^{2}\right]\right\}-\frac{1}{12} \rho \alpha G_{x}+(1-\alpha)\left[N_{7}\left(z_{2}\right)\right.
\end{aligned}
$$

$$
\begin{aligned}
&-1 / \tau\left\{N_{7}\left(z_{2}\right)-\frac{1}{36} \rho\left[1-3 u_{x}\left(z_{2}\right)+3 u_{x}\left(z_{2}\right)^{2}\right]\right\} \\
&\left.\left.-\frac{1}{12} \rho G_{x}\right]\right), \\
& N_{6}\left(z_{1}\right)=\alpha\left(N_{8}\left(z_{1}\right)-1 / \tau\left\{N_{8}\left(z_{1}\right)-\frac{1}{36} \rho \alpha\left[1+3 u_{x}\left(z_{1}\right)\right.\right.\right. \\
&\left.\left.+3 u_{x}\left(z_{1}\right)^{2}\right]\right\}+\frac{1}{12} \rho \alpha G_{x}+(1-\alpha)\left[N_{8}\left(z_{2}\right)\right. \\
&-1 / \tau\left\{N_{8}\left(z_{2}\right)-\frac{1}{36} \rho\left[1+3 u_{x}\left(z_{2}\right)+3 u_{x}\left(z_{2}\right)^{2}\right]\right\} \\
&+\left.\left.\frac{1}{12} \rho G_{x}\right]\right), \\
& N_{6}\left(z_{2}\right)= N_{6}\left(z_{1}\right)-1 / \tau\left\{N_{6}\left(z_{1}\right)-\frac{1}{36} \rho \alpha\left[1-3 u_{x}\left(z_{1}\right)\right.\right. \\
&\left.\left.+3 u_{x}\left(z_{1}\right)^{2}\right]\right\}-\frac{1}{12} \rho \alpha G_{x}+\frac{1-\alpha}{\alpha} N_{6}\left(z_{1}\right) . \\
& N_{7}\left(z_{1}\right)= \alpha\left[N_{7}\left(z_{2}\right)-1 / \tau\left\{N_{7}\left(z_{2}\right)-\frac{1}{36} \rho\left[1-3 u_{x}\left(z_{2}\right)\right.\right.\right. \\
&\left.\left.\left.+3 u_{x}\left(z_{2}\right)^{2}\right]\right\}-\frac{1}{12} \rho G_{x}\right], \\
& N_{3}\left(z_{2}\right)= \frac{1}{9} \rho\left[1-3 u_{x}\left(z_{2}\right)+3 u_{x}\left(z_{2}\right)^{2}\right]-\frac{1}{3} \rho \tau G_{x}, \\
& N_{1}\left(z_{2}\right)= \frac{1}{9} \rho\left[1+3 u_{x}\left(z_{2}\right)+3 u_{x}\left(z_{2}\right)^{2}\right]+\frac{1}{3} \rho \tau G_{x}, \\
&\left.\left.+3 u_{x}\left(z_{2}\right)^{2}\right]\right\}+\frac{1}{12} \rho \alpha G_{x}+\frac{1-\alpha}{\alpha} \rho G_{5}\left(z_{1}\right), \\
& N_{8}\left(z_{1}\right)= \alpha\left[N_{8}\left(z_{2}\right)-1 / \tau\left\{N_{8}\left(z_{2}\right)-\frac{1}{36} \rho\left[1+3 u_{x}\left(z_{2}\right)\right.\right.\right. \\
&
\end{aligned}
$$

Distributions $N_{7}\left(z_{2}\right)$ and $N_{8}\left(z_{2}\right)$ are kept as unknown. Substitution of the above equations in the definition of the velocities at $z_{1}$ and $z_{2}$ of Eq. (9) results in two equations which only contain the unknown $N_{8}\left(z_{2}\right)-N_{7}\left(z_{2}\right)$ term and the velocities $u_{x}\left(z_{1}\right)$ and $u_{x}\left(z_{2}\right)$. By combining these two equations and thereby removing the term $N_{8}\left(z_{2}\right)-N_{7}\left(z_{2}\right)$, Eq. (10) is obtained. 
[1] S. Chen and G.D. Doolen, Annu. Rev. Fluid Mech. 30, 329 (1998).

[2] D. Kandhai, U. Tallarek, D. Hlushkou, A.G. Hoekstra, P.M.A. Sloot, and H. van As, Proc. R. Soc. London, Ser. A 360, 521 (2002).

[3] J.J. Derksen and H.E.A. van den Akker, AIChE J. 45, 209 (1999).

[4] D. Kandhai, A. Koponen, A.G. Hoekstra, M. Kataja, J. Timonen, and P.M.A. Sloot, J. Comput. Phys. 150, 482 (1999).

[5] P. Skordos, Phys. Rev. E 48, 4823 (1993).

[6] T. Inamuro, M. Yoshino, and F. Ogino, Phys. Fluids 7, 2928 (1995).

[7] D. Noble, J. Georgiadis, and R. Buckius, Int. J. Numer. Methods Fluids 23, 1 (1996).

[8] I. Ginzbourg and D. d'Humiéres, J. Stat. Phys. 84, 927 (1996).

[9] R. Maier, R. Bernard, and D. Grunau, Phys. Fluids 8, 1788
(1996).

[10] S. Chen, D. Martinez, and R. Mei, Phys. Fluids 8, 2527 (1996).

[11] R. Verberg and A. Ladd, Phys. Rev. E 65, 016701 (2001).

[12] H. Chen, C. Teixeira, and K. Molvig, Int. J. Mod. Phys. C 9 , 1281 (1998).

[13] M. Bouzidi, M. Firdaouss, and P. Lallemand, Phys. Fluids 13, 3452 (2001).

[14] H. Chen, Phys. Rev. E 58, 3955 (1998).

[15] X. He, Q. Zou, L.-S. Luo, and M. Dembo, J. Stat. Phys. 87, 115 (1997).

[16] N. Cao, S. Chen, S. Jin, and D. Martinez, Phys. Rev. E 55, R21 (1997).

[17] I. Karlin, S. Succi, and S. Orszag, Phys. Rev. Lett. 82, 5245 (1999).

[18] M. Rohde, J.J. Derksen, and H.E.A. van den Akker, Phys. Rev. E 65, 056701 (2002). 